\title{
Análisis motivacional del uso del smartphone entre jóvenes: una investigación cualitativa
}

\author{
Ubaldo Cuesta CAmbra \\ Universidad Complutense de Madrid \\ ucuestac@ucm.es \\ Sandra GASPAR HERRERo \\ Universidad Complutense de Madrid \\ sgasparherrero@ucm.es
}

\begin{abstract}
Resumen
El smartphone se ha convertido en un objeto imprescindible en la vida diaria de los adolescentes, provocando en ocasiones cierto uso problemático o adictivo (Chóliz 2012). Esta investigación analiza el imaginario colectivo que subyace a esta conducta con el objetivo de conocer sus bases motivacionales. Los resultados parecen indicar que existe, bajo determinadas condiciones, un elevado riesgo de "conducta excesiva".
\end{abstract}

Palabras clave: Adicción móvil - Redes Sociales - Psicología comunicación - Smartphone

\section{Motivational analysis of smartphone use among young people: a qualitative research}

\begin{abstract}
The smartphone has become a must-have item in the daily lives of teenagers, sometimes causing some problematic or addictive use (Chóliz 2012). This research analyzes the collective imagination behind this behavior in order to meet their motivational bases. The results suggest that there is, under certain conditions, a high risk of "excessive behavior".
\end{abstract}

Keywords: Mobile addiction-Social networks -Psychology of communication-smartphone.

Referencia normalizada:

Cuesta Cambra, U.; Gaspar Herrero, S. (2013) Análisis motivacional del uso del smartphone entre jóvenes: una investigación cualitativa. Historia y Comunicación Social. Vol. $18 \mathrm{~N}^{\circ}$ Especial Noviembre. Págs. 435-447.

Sumario: 1.Introducción. 2. Marco teórico. $2.1 \mathrm{El}$ "Teléfono inteligente" o Smartphone. 2.2 Uso abusivo de las tecnologías. 3.1 Objetivos e hipótesis. 3.1 Objetivos. 3.2 Hipótesis. 4. Metodología. 4.1. Composición de las entrevistas en profundidad y de los grupos de discusión (GD). 5. Resultados. 5.1. Análisis del discurso. 6. Conclusiones. 7. Bibliografía.

\section{Introducción}

En la actualidad el empleo del smartphone o teléfono inteligente (TI) se ha transformado en un instrumento imprescindible en la vida cotidiana de muchos adolescen- 
tes. Según algunos autores como Chóliz (2012) se trata de una verdadera adicción dado que cumple los criterios indicados para su diagnóstico: síndrome de abstinencia, provocación de conflictos personales, interferencia con otras actividades, etc. (Brown, 1993; APA, 2000).

La presente investigación analiza el "imaginario colectivo" que subyace a esta conducta, para intentar conocer las bases motivacionales profundas que subyacen a la misma.

En otros trabajos (Cuesta, 2012) se ha investigado desde una perspectiva psicométrica este fenómeno, proporcionando las bases para desarrollar un cuestionario de evaluación del "uso envolvente del TI". En este trabajo, mediante una metodología cualitativa (Elliott, Fischer, Rennie, 1999), se ha profundizado en las motivaciones psicosociales que subyacen al fenómeno de estudio (Walsh, White, Young, 2008).

Algunos autores han investigado la adicción al TI mediante la metodología cualitativa (Lapointe, Boudreau-Pinsonneault y Vaghefi, 2013) aunque existe muy poca evidencia empírica rigurosa en este sentido. Mediante focus group (Morgan, 1998) se estudiaron las motivaciones profundas de los adolescentes para el empleo "envolvente" del TI; así mismo se estudiaron las experiencias y opiniones de padres al respecto.

Por último se han realizado entrevistas en profundidad (Callejo, 2002) a expertos del sector con importantes conocimientos y experiencias sobre el tema bajo los criterios: 1. Ejercen profesiones relacionadas con estas tecnologías, 2. Trabajan en patologías asociadas al empleo de las TIC y 3. Están en contacto directo con las necesidades actuales de formación en las TIC.

En todos los casos, las muestras fueron seleccionadas según los criterios de muestreo propios de la investigación.

\section{Marco teórico}

\subsection{El "teléfono inteligente" o smartphone}

Al hablar de teléfonos móviles, con frecuencia, nos referimos a los llamados "teléfonos inteligentes". Este tipo de dispositivos, presentan algunas características muy especiales que les hacen especialmente sensibles para provocar conductas problemáticas o adictivas. Entendemos por uso problemático aquel que presenta patrones muy similares a aquellos que se encuentran en conductas adictivas (Brown, 1993). Las características que pueden provocar este tipo de comportamientos se basan fundamentalmente en dos variables:

- Transporte muy cómodo y siempre se encuentran en disposición de uso. 
- Conexión con Internet instantánea, de muy fácil acceso y rápida a precio de "tarifa plana". Se trata de un precio muy asequible que ha hecho que una gran cantidad de jóvenes dispongan actualmente de este tipo de tarifa.

\subsection{Uso abusivo de las tecnologías}

La adicción a las nuevas tecnologías, y más concretamente al TI está apoyada en diversos aspectos psicológicos muy complejos y muy adictivos.

En primer lugar, es necesario considerar la importancia de las redes sociales en este contexto. Como es sabido, los TI permiten estar constantemente conectados al grupo de amigos, familiares, y todo un conjunto de entornos sociales muy importantes para la persona; y todavía más, permite estar pendientes de todas las actividades de tipo social que se están realizando a mí alrededor. De esta manera, las redes sociales inciden sobre dos de los aspectos más importantes desde el punto de vista de la motivación social.

Por un lado, el motivo social de "búsqueda de información". Consiste en la necesidad imperiosa por parte de los sujetos de estar constantemente buscando información en su entorno. Por otro lado, suele asociarse a esta motivación social el deseo de "búsqueda de contactos". Se trata de la motivación social que empuja a las personas a buscar relaciones sociales entre ellas. Esta motivación social es especialmente importante en la adolescencia, en la cual se produce el paso de las relaciones familiares a la relaciones de amistad. Este es, por lo tanto, uno de los aspectos más importantes que subyacen a la adicción del TI.

De esta manera, recientemente, se ha comenzado hablar del síndrome de FOMA: fear of missing out (Przybylski, Murayama, Dehaan, Gladwell, 2013). Se trata de "miedo a sentirse fuera": miedo a perder el contacto con los demás y con las actividades que estos organizan. Las discusiones de grupo cualitativo, nos ayudarán a esclarecer y profundizar en este punto.

\section{Objetivos e hipótesis}

\subsection{Objetivos}

El objetivo principal de esta investigación ha consistido en analizar los mecanismos motivacionales profundos que subyacen al empleo del TI. Se han desarrollado los siguientes objetivos específicos:

- Conocer las actitudes y experiencias de utilización de los smartphones que expliquen el "por qué" se conectan.

- Identificar los mecanismos que facilitan la utilización de los smartphones, con especial hincapié en la accesibilidad y disponibilidad. 
- Conocer las ventajas y desventajas en la influencia de la utilización de los smartphones en los procesos de interacción personal de los adolescentes; concretamente en las dimensiones de las relaciones personales, sociales y familiares, centrándonos

- Profundizar en los mecanismos propios de los hábitos de empleo del IT.

- Determinar qué formas de utilización de los smartphones pueden derivar en una conducta adictiva por parte de los adolescentes.

Se trata, de cinco objetivos de análisis: 1.Por qué se conectan, 2.Influencia de la accesibilidad y disponibilidad, 3. Ventajas y desventajas percibidas, 4. Hábitos de conexión y 5 . ¿Conducta adictiva?

\subsection{Hipótesis}

- H1: Los smartphones se han convertido en la herramienta por excelencia preferida por los adolescentes en la búsqueda de "nuevas sensaciones" (sensation seeking).

- H2: El aumento del empleo del smartphone entre los jóvenes se debe en gran parte a su accesibilidad instantánea y a su disponibilidad: los jóvenes lo utilizan durante todo el día, en cualquier momento y hora convirtiéndose en imprescindibles para relacionarse con sus grupos de iguales y familiares. El constructo psicológico "control de impulsos" (Pedrero y cols., 2007).

- H3: Los jóvenes aprecian determinadas ventajas del uso del smartphone como pueden ser: uso en caso de emergencia, estar siempre localizable por los amigos. Sin embargo también observan ciertas desventajas como puede ser el control parental excesivo y problemas ocasionados por la pérdida de privacidad.

- H4: Los jóvenes emplean los smartphones en su vida diaria común: clase, en casa, actividades de ocio, etc. El smartphone "envuelve" todas las dimensiones o esferas de la vida del adolescente. Es posible que se puedan distinguir diferentes "patrones de uso excesivo" o tipologías.

- H5: La excesiva facilidad que los adolescentes tienen para acceder y utilizar diariamente estas tecnologías, así como su aparente "indispensabilidad" entre el propio grupo de amigos puede influir en la génesis de una conducta excesiva de uso. Síndromes como el FOMO pueden responder a esta motivación. 


\section{Metodología}

Se ha basado en la realización de investigación cualitativa (Elliott, Fischer, Rennie, 1999), desarrollada mediante técnicas de las entrevistas en profundidad (Callejo, 2002) y grupos de discusión (Morgan, 1998).

La técnica de investigación en profundidad se aplicó a los expertos. Con padres y madres de adolescentes y con los propios jóvenes y adolescentes usuarios de smartphones se empleó la técnica del focus group (Morgan, 1998).

4.1 Composición de las entrevistas en profundidad y de los grupos de discusión (GD).

La selección de los participantes se ha realizado mediante un muestreo intencionado. Se han buscado expertos profesionales que desempeñen tareas profesionales relacionadas con tecnologías pensadas para población de franjas de edad adolescente-juvenil. Se realizaron 6 entrevistas en profundidad.

La formación de los grupos de discusión, se configuró atendiendo a los siguientes criterios de inclusión.

En el GD1 los criterios fueron: Edad: de 14 a 16 años (50\% de cada sexo). En el GD2: de 18 a 20 años (50\% de cada sexo). Nivel de estudios: 50\% no universitarios y $50 \%$ universitarios. En el GD3: Padres que tienen hijos adolescentes, usuarios de Smartphone. Sexo: $50 \%$ de cada sexo.

\section{Resultados}

\subsection{Análisis del discurso}

Se exponen los resultados encontrados agrupados por categorizaciones de las hipótesis y especificando si pertenece al grupo de discusión (GD1, 2 ó 3) o a la entrevista con expertos (EP).

\subsection{1. ¿Por qué se conectan?}

El "gancho" de los smartphones no es tanto la herramienta en sí misma, sino lo que supone usar esa herramienta en concreto. La adolescencia es una etapa de la vida en la que el proceso de socialización de la persona adquiere especial importancia, por ello el papel de la comunicación con las personas que integran el entorno es clave. La utilidad principal de los smartphones para los adolescentes es, pues, facilitar esa comunicación.

"El gancho es que puedes estar en contacto permanentemente". GD3 
"(...) el gancho es el WhatsApp". GD2

"(...) poder estar informado de lo que pasa en el mundo". EP GD3

“Es que le sacan un partido al teléfono, (...) para todo, música, videos de Youtube.

Con la comunicación se posibilita su participación activa en la formación de una red de relaciones sociales en las que el mayor peso es el otorgado a sus iguales, los amigos y la "pandilla".

"El gancho es poder vacilar con los amigos". GD3.

"Es una forma de quedar de la pandilla, de saber qué pasa, de socialización de los jóvenes". EP

"Compartir fotos con tus amigos. Comunicación instantánea. Interactuar en tiempo real y comunicarse". EP

Otro segundo uso importante del móvil es para poder contactar con los conocidos de sus amigos. De esta manera se amplía el número de "amigos" que se pueden llegar a tener por los amigos de otros amigos. Finalmente se conectan para seguir también las acciones y opiniones de sus "líderes de opinión" o de sus "modelos mediáticos". Así se va ampliando de modo online el círculo de sus relaciones sociales.

“También es una forma para estar en contacto con mis amigos, con mi familia y también un poco las noticias que hay, sobre las páginas que me interesan, estar conectada todo el tiempo". GD2.

"Se siguen a los "ídolos", futbolistas si son de deporte,... seguir citas de famosos y retuitearlas, ... fotografías como Instagram". EP

El adolescente tiene prisa por cerrar la puerta de la etapa de la niñez, donde el control familiar es más fuerte y abrir la que les introduce en la edad adulta, sinónimo de mayor libertad de acción y alejamiento del control paternal. En opinión de los padres (GD3):

“(...) el teléfono siempre va en silencio.

"Muchas veces no te cogen el teléfono pero si contestan los WthatsApp.(...) eso es una condena. Los llamas para algo y nunca te lo cogen".

Por consiguiente, se confirma la hipótesis de que los jóvenes se conectan para "buscar sensaciones" pero se precisa que éstas son de tipo social. Es decir, se podría hablar de un patrón de "búsqueda de sensaciones sociales" (social sensation seeker).

\subsubsection{Accesibilidad y disponibilidad}

Los jóvenes son conscientes de la facilidad con que pueden acceder a estos recursos, la oportunidad de disponibilidad que se les ofrece. El móvil se sitúa 
de tal forma en el centro del engranaje que integran estas tecnologías que ninguno de los informantes puede ser capaz de identificar a algún joven que no disponga de esta herramienta.

"Cada vez se nos ofrecen mejores tablets, móviles más polivalentes, gadgets más baratos y esto hace que la gente quiera tener el último móvil”. EP

"Yo creo que nos hemos acostumbrado a las facilidades y las cosas rápidas". GD2.

"Tengo uno (ordenador) en mi habitación, otro en el salón, y luego tablets y Play (...), para no aburrirnos". GD1.

Esta accesibilidad potencia las dificultades de control de impulsos detectadas en conductas vinculadas al sistema de refuerzos inmediatos (Pedrero y cols., 2007). Se confirma, pues, la hipótesis, pero es necesario profundizar más en la importancia que supone el "libre acceso" a estas conductas debido a: (1) las tarifas planas, (2) la accesibilidad del smartphone (que se lleva siempre consigo).

\subsubsection{Ventajas frente a desventajas}

La principal desventaja que identifican todos los informantes es la pérdida de privacidad. Es la primera barrera que identifican sobre todo los padres, denostando el peligro que supone la conexión permanente. Es un determinante de la desconfianza que manifiestan hacia la utilización "abusiva" de los smartphones, por parte de sus hijos. La falta de privacidad es percibida, en ocasiones, como pérdida de la intimidad familiar. Otras veces, sobre todo para los padres que tienen hijas, deriva en un sentimiento de preocupación, de miedo a que sus hijas puedan llegar a estar en peligro. Es la sensación de que hay otras personas que lo saben todo de sus actividades, de sus costumbres, de sus horarios.

"Dan mucha información de ti. La ley de protección es muy fácil saltársela". GD3.

"A mi hija me da más cosa y se lo digo, no pongas fotos en bañador, mira lo que pasa en las noticias (...) y si algún día te ocurre, tú nos lo cuentas. GD3.

"Depende de cómo lo uses. Si pones cosas íntimas, personales, en las redes sociales, te la estás jugando". GD 2.

"En el momento en que cuelgas algo en la red deja de ser privado. Eres tú mismo el que tienes que ver qué cuelgas y qué no, por muchas trabas que pongas". EP

La esfera de lo privado se empequeñece a medida que aumenta la utilización de estas herramientas de comunicación. La información personal sobre las actividades que se hacen en cada momento, todas las opiniones, conversaciones a través de mensajes, las fotografías o vídeos que se realizan con motivo de acontecimientos y celebraciones privadas pasan a convertirse en algo público. Los adolescentes se mueven en un entramado en el que se les anima constantemente a revelar cuanta más información mejor. 
"Hacer fotos (una amiga) a un niño de meses todos los días y que lo suba a 400 amigos. Es como el show de Truman, todo el mundo se sabe todo (...) Subir fotos de todo a todas horas, nos estamos descontrolando un poco". EP

"Es que te llega (una foto) y tú no tienes la opción de no aceptarla...y esa foto puede ir rulando por ahí..." GD2.

"Es lo que ha pasado con un par de chicas que han subido fotos y videos muy ,muy íntimos, y se lo han ido pasando por toda España. GD2.

“Yo creo que si tienes una foto más comprometida la puedes subir al Facebook y poner que la vean solo tus amigos, pero si la foto está en internet y es más comprometida o algo personal que no quieres que vea nadie, yo por lo menos no lo subiría." GD2.

"Hay gente que lo cuenta todo: "estoy saliendo" "he comprado esto" (...) eso es ser ya estúpido".GD1.

La hipótesis ha sido sólo parcialmente confirmada: aparece como una de las desventajas más señaladas por los jóvenes el problema de la "intimidad" y el acceso a ella por outsiders indeseados.

\subsubsection{Hábitos de conexión}

Cuando se les pregunta cuántas veces al día y cuánto tiempo usan el móvil, los jóvenes y adolescentes responden: "Constantemente, todo el día”. La utilización del móvil se ha convertido en un hábito diario para ellos. De lunes a viernes y los fines de semana. Se chequea el móvil incluso sabiendo que ha transcurrido poco tiempo para que hayan llegado mensajes nuevos y aunque la pantalla no se haya iluminado, por si acaso. GD2

"Uso el móvil todos los días principalmente para el WhatsApp y luego llamar.

Llamo sobre todo los fines de semana, cuando los tengo gratis y mensajes muy poquitos. Y luego me meto al Tuenti, al Facebook (...)".GD1

“Mis padres me dicen que no pase tanto tiempo con el móvil, (...) no sé.”GD2

Los padres identifican algunos factores que pueden contribuir a la aparición de obstáculos que entorpecen este programa educacional. Uno de ellos es el problema con la conciliación laboral y familiar.

"En este país la conciliación laboral y la familiar no casan. A mí me gustaría levantarme a las 5 o a las 6 para tener las tardes libres y poder pasarla con mis hijos. Como en las películas americanas, yo les envidio." GD3

En cualquier caso mi hija sale a las 5:30 del cole y llega a casa las 6.(..) sin lavarnos las manos, ni merendar, ni nada, dos horas de deberes. ¿De dónde sacas más horas? ¿Dónde vas con ellos a esas horas? Ya es de noche.”GD3. 
Otro el de "las malas" influencias de ciertos contextos familiares que predisponen a que los adolescentes hagan un uso inadecuado.

"No puedes utilizar a tu hijo para que coma con tu móvil (...) no se puede decir la culpa es de las redes sociales." EP

"Lo mío es peor, mi hija vive con el WhatsApp y con el Tuenti desde que se levanta, pero es que su padre le pasa lo mismo. Le regaña a ella pero él es peor." GD3.

Para saber hasta qué punto controlan los jóvenes el uso de su móvil y si demuestran suficiente madurez a la hora de utilizarlo. La respuesta es que la mayoría de los jóvenes sí que se ven maduros $y$, por supuesto, controlando; pero parece ser que a la hora de hablar de madurez y control todo dependerá de cómo sea cada persona.

"En líneas generales los jóvenes demuestran responsabilidad; pero depende de la edad. Los adolescentes de 14 a 18 años, la mayoría hace un uso responsable". EP

"Es más fácil ser un inmaduro al usar un móvil que lo que se es en la vida real".EP

\subsection{5. ¿Momento de hablar de adicción o dependencia?}

Los adolescentes pierden horas de sueño para subir una foto en internet; se pueden olvidar las llaves antes de salir de casa, pero nunca se olvidan el móvil. Para la realización de ciertas actividades diarias aumenta la sensación de seguridad si llevan el TI encima; cunde la histeria cuando se les estropea el móvil; necesidad de jugar una partida todos los días antes de ir al trabajo; sensación de libertad cuando llegan las vacaciones y pueden olvidarse de él.

"Ya en nuestra época (la de los padres), nos decían "estás enganchado al teléfono. El usar el teléfono es para cosas importantes".GD3

"Que alguien procese su cabeza a las 3 de la mañana subir al Tuenti o al Facebook una foto... que se ponga a etiquetar es duro", eso sí que es muy adictivo (estoy en los cines, estoy en (...) y manda una foto)." GD3

"Como se rompa el móvil de un hijo la que se arma en casa es brutal. No pueden vivir sin él (...) drogadicción en vena”.GD3

"El mono.”GD3

"Yo me acuerdo una vez que hubo como un fallo en la red de WhatsApp o algo así, hace un año, y estuvo tres días sin funcionar, y yo creo que casi se acababa el mundo. O sea, si que dependemos mucho. Yo creo que es malo".GD3

Las siguientes respuestas son para comprender por qué ese uso se ha ido convirtiendo en algo tan necesario en su vida diaria; o de si en la búsqueda de un culpable, es el móvil una tecnología que favorece la adicción o son algunos jóvenes los que poseen una personalidad adictiva; y por ultimo de si esto no significa que se ha 
llegado a un punto en el que debería de empezar a hablarse taxativamente de dependencia, o de adicción, o de abuso por parte de los jóvenes y adolescentes con respecto a la utilización de los móviles. La percepción de los padres se inclina por dar una respuesta afirmativa.

"Lo que tienen los chicos es vicio". GD3.

"Hay que saber desconectar, y ellos no lo saben." GD3.

"Yo pienso que al principio era una innovación y que estaba bien pero ahora nos estamos volviendo adictos. Si nos quitan el WhatsApp, desde mi punto de vista no sabríamos vivir sin él.”GD2.

Hay que continuar indagando por si aparece en el discurso la descripción de algunos síntomas que son característicos de la mayoría de los tipos de adicciones y que puedan hacer sospechar de la existencia de ésta. Se encuentra entonces un amplio abanico de descripciones: se pierden horas de sueño para subir una foto en internet; se pueden olvidar las llaves antes de salir de casa, pero nunca se olvida el móvil; para la realización de ciertas actividades diarias aumenta la sensación de seguridad si se lleva encima; cunde la histeria cuando se les estropea el móvil; necesidad de jugar una partidita todos los días antes de ir al trabajo; sensación de libertad cuando llegan las vacaciones y te olvidas de él

"Ya en la nuestra, época (la de los padres), nos decían "estás enganchado al teléfono. El usar el teléfono es para cosas importantes"." EP

"Que alguien procese su cabeza a las 3 de la mañana subir al Tuenti o al Facebook una foto...que se ponga a etiquetar es duro", eso sí que es muy adictivo (estoy en los cines,estoy en (...) y manda una foto)." EP

"En mi casa tengo un altar y pongo velas. Dios mío que no se les estropee el móvil, que no se les rompa". GD3

"Se ponen histéricos si se les estropea (...) andan histéricos por la casa.." GD3

"El mono" GD3.

"Yo me acuerdo una vez que hubo como un fallo en la red de WhatsApp o algo así, hace un año, y estuvo tres días sin funcionar, y yo creo que casi se acababa el mundo. O sea, si que dependemos mucho. "Yo creo que es malo." GD2.

\section{Conclusiones}

El análisis de los discursos extraídos de los focus group y de las entrevistas en profundidad realizadas a los expertos, permiten responder a las hipótesis planteadas y extraer, en síntesis, las siguientes conclusiones: 
Los smartphones son, por la cantidad de atractivos y recompensas que ofrecen para los jóvenes, una herramienta con un potencial "enganche" que hay que tener en cuenta. El smartphone es empleado por los jóvenes no solo como una herramienta de comunicación (llamadas, mensajería, internet), si no que ha reemplazado a otros gadgets como son: despertadores o alarmas, cámaras (fotográficas y de video), agendas (de papel o electrónicas). (Walsh, White, Young, 2008).

Los smartphones se han convertido en un objeto con presencia continúa en los hábitos diarios de los jóvenes: en casa, en clase, en los momentos de ocio (Lorente, 2002). Es por ello que no sorprende el extremo "apego" o "engagement" de los jóvenes hacia su teléfono móvil. Se ratifican las hipótesis sobre la "búsqueda de sensaciones por parte de los adolescentes", así como del "uso envolvente del smartphone".

La accesibilidad y disponibilidad de los smartphones se ha normalizado de tal manera que es casi imposible encontrar un adolescente o joven que no mantenga contacto con esta tecnología. El proceso de socialización de los jóvenes no puede ya concebirse sin la utilización de los smartphones, así como el contacto, relación y en algunos casos imitación de ciertos líderes sociales (bien sean cercanos, dentro de su grupo de amigos, o de la sociedad general). Para los jóvenes, la vida, en particular, y el mundo en general, serían demasiado aburridos sin la presencia de los smartphones. Se confirma la hipótesis sobre la construcción de avatares por imitación de "modelos mediáticos" o "líderes" en su grupo de relaciones y que la misma en ciertos casos, derive en un uso inadecuado y en ocasiones adictivo.

Respecto a las ventajas que ofrecen los smartphones se destaca que favorecen el proceso de socialización de los jóvenes, favoreciendo la comunicación (gracias a la rapidez e inmediatez que permite), así como su alta disponibilidad de uso en cualquier lugar o momento. Se confirma la hipótesis sobre la facilidad que los adolescentes tienen para acceder y utilizar diariamente estas tecnologías.

Por otro lado, aparecen también inconvenientes del uso del smartphone como es la pérdida de privacidad o de intimidad familiar; produciéndose a su vez un cambio en las formas de interacción y relación social, que en ocasiones se percibe como perjudicial: aislamiento del entorno, deterioro de las relaciones familiares, detrimento de formas de expresión oral y escrita, e incluso transformación de las actividades de ocio. Se confirma la hipótesis sobre la utilización inadecuada de los smartphones que deforma la percepción de los adolescentes y favorecer la elaboración de una realidad social virtual.

Existe un importante salto generacional con respecto a las estrategias de uso, para comprender las actitudes y reacciones de los padres. Los smartphones, por sí mismos, no son buenos ni malos. Por ello adquiere una gran relevancia la educación de los jóvenes para que hagan un uso inteligente y saludable de estos móviles; aparece la necesidad de educación familiar específica con respecto al uso del smartphone, así como de educación institucional. 
Por consiguiente, como Cassidy sugiere (2006), el uso del móvil es una "adicción positiva" en las cual existen beneficios derivados del propio comportamiento de uso del smartphone (Glasser, 1985). Sin embargo, Orford (2001) advierte que las "adicciones positivas" pueden convertirse en "adicciones negativas" cuando consecuencias adversas (como el síndrome de abstinencia), superan los beneficios del comportamiento.

Existe un consenso general respecto a que la adicción al smartphone puede aparecer y los usuarios identificar ciertos comportamientos compulsivos y síntomas del uso abusivo como: chequeo continúo de mensajes o llamadas, o incompatibilidad con la realización de actividades cotidianas. Autores como Charlton y Danforth (2007) distinguen entre "comportamiento adictivo", respecto a "comportamiento excesivo" en relación con el uso a internet o al juego online. Queda confirmada la hipótesis sobre la aparición de conductas adictivas como consecuencia del uso excesivo del smartphone.

Algunos participantes asumen que la adicción al smartphone puede existir, pero no la perciben como una adicción tan negativa como pudiera ser el consumo de sustancias ilegales o el tabaco.

La posibilidad de riesgo de hacer un uso inadecuado o adictivo, depende más de las características internas del propio joven que de la herramienta en sí misma.

Finalmente, analizando los resultados de los topis y Las hipótesis 4 y 5 podría concluirse que parecen existir diferentes patrones de uso en una conducta de uso excesivo: sujetos con ansiedad por la pérdida de acontecimientos externos (FOMO), sujetos muy socializados pero sin ese componente ansioso y sujetos buscadores de sensaciones constantes (compulsivas). Futuras investigaciones deberán profundizar en estas tipologías.

\section{Bibliografía}

BIANCHI, A., \& PHILLIPS, J. G. (2005)."Psychological predictors of problem mobile phone use". En: Cyber Psychology and Behavior, $\mathrm{n}^{\circ}$ 8, pp.39-51.

CALLEJO, J. (2002). "Observación, entrevista y grupo de discusión: el silencio de tres prácticas de investigación”. En: Revista Española de Salud Pública, n5, pp. 409-422.

CASSIDY, S. (2006). "Using social identity to explore the link between a decline in adolescent smoking and an increase in mobile phone use". En: Health Education, $\mathrm{n}^{\mathrm{o}} 106$, pp. $238-250$.

CUESTA, Ubaldo (2013): "Uso "envolvente" del móvil en jóvenes: propuesta de un modelo de análisis"..En: Estudios sobre el mensaje periodístico, nº 18, pp.253-262.

CHARLTON, J. \& DANFOTH, I. (2004).'Distinguishing addiction and high engagement in the context of online game playing". En Computers Human Behavior, n²3, pp. Pages 1531-1548 
CHÓLIZ, M (2012). "Mobile-phone addiction in adolescence: The Test of Mobile Phone Dependence (TMD)". En: Progress in Health Sciences, $\mathrm{n}^{\circ} 1, \mathrm{pp} .33-44$.

ELLIOTT, R., FISCHER, C. T., \& RENNIE, D. L. (1999).”Evolving guidelines for the publication of qualitative research studies in psychology and related fields". En: The British Journal of Clinical Psychology, $\mathrm{n}^{\mathrm{o}}$ 38, pp.215-229.

LING, R., \& HELMERSEN, P. (2000): It must be necessary; it has to cover a need: The adoption of mobile telephony among pre-adolescents and adolescents. Presentado en "Social Consequences of Mobile Telephony", Oslo (Noruega).

MORGAN, D. L. (1998). Planning focus groups (libro 2) focus group kit. Thousand Oaks, CA: Sage.

MURPHY, E., \& DINGWALL, R. (2003). Qualitative methods and health policy research. Nueva York: Walter de Gruyter.

ORFORD. (2001). Excessive appetites: A psychological view of addictions (2nd ed.). Chichester, UK: Wiley.

PRZYBYLSKI, Andrew K. MURAYAMA, Kou, DEHAAN, Cody R. \& GLADWELL, Valerie (2013). "Motivational, emotional, and behavioral correlates of fear of missing out". En: Computers in Humarn Behavior, n²9, pp. 1841-1848.

SRIVASTAVA, L. (2005). "Mobile phones and the evolution of social behavior". En: Behaviour and Information Technology, $\mathrm{n}^{\circ} 24$.

WALSH, S., WHITE, K. \& YOUNG, R. (2008). "Over-connected? A qualitative exploration of the relationship between Australian youth and their mobile phones". En: Journal of Adolescence, $\mathrm{n}^{\circ} 31, \mathrm{pp} .77-92$.

\section{Los autores}

Ubaldo Cuesta Cambra es Doctor en Psicología de la Comunicación y Catedrático de la Universidad Complutense de Madrid.

Sandra Gaspar Herrero es Doctoranda en Psicología de la Comunicación en la Universidad Complutense de Madrid. 